\title{
Corrigendum: Atomically resolved imaging of highly ordered alternating fluorinated graphene
}

Reza J. Kashtiban, M. Adam Dyson, Rahul R. Nair, Recep Zan, Swee L. Wong, Quentin Ramasse, Andre K. Geim, Ursel Bangert \& Jeremy Sloan

Nature Communications 5:4902 doi: 10.1038/ncomms5902 (2014); Published 16 Sep 2014; Updated 12 Nov 2014

In the Methods section of this Article, the details of the STEM equipment and operating parameters used for the EELS studies were provided incorrectly. The following are the correct details: 'EELS studies were also performed on partially fluorinated graphene samples at the SuperSTEM Laboratory on a Nion UltraSTEM100 dedicated ultrahigh vacuum scanning transmission electron microscope equipped with a cold field emission gun with a native energy spread of $0.3-0.35 \mathrm{eV}$ and operating at $60 \mathrm{keV}$.' 